\title{
Globulelike Conformation and Enhanced Diffusion of Active Polymers
}

\author{
Valentino Bianco, ${ }^{1,2, *}$ Emanuele Locatelli, ${ }^{1, \dagger}$ and Paolo Malgaretti ${ }^{3,4, \$}$ \\ ${ }^{1}$ Faculty of Physics, University of Vienna, Boltzmanngasse 5, Wien 1090, Austria \\ ${ }^{2}$ Faculty of Chemistry, Chemical Physics Deprtment, Universidad Complutense de Madrid, Plaza de las Ciencias, \\ Ciudad Universitaria, Madrid 28040, Spain \\ ${ }^{3}$ Max Planck Institute for Intelligent Systems, Heisenbergstrasse 3, 70569 Stuttgart, Germany \\ ${ }^{4}$ Institute for Theoretical Physics IV, University of Stuttgart, Pfaffenwaldring 57, 70569 Stuttgart, Germany
}

(Received 28 May 2018; published 21 November 2018)

\begin{abstract}
We study the dynamics and conformation of polymers composed by active monomers. By means of Brownian dynamics simulations we show that, when the direction of the self-propulsion of each monomer is aligned with the backbone, the polymer undergoes a coil-to-globulelike transition, highlighted by a marked change of the scaling exponent of the gyration radius. Concurrently, the diffusion coefficient of the center of mass of the polymer becomes essentially independent of the polymer size for sufficiently long polymers or large magnitudes of the self-propulsion. These effects are reduced when the self-propulsion of the monomers is not bound to be tangent to the backbone of the polymer. Our results, rationalized by a minimal stochastic model, open new routes for activity-controlled polymers and, possibly, for a new generation of polymer-based drug carriers.
\end{abstract}

DOI: 10.1103/PhysRevLett.121.217802

Diverse biological systems feature chemical reactions and energy conversion occurring on the backbone of polymers, often involving active components. For example, DNA is duplicated by DNA-polymerase actively displacing on it [1]; ribosomes synthesize proteins by actively sliding along RNA strands [1,2]. Synthetic realizations of such processes hint at intriguing applications for microdevices and nanomedicine [3-6]. The current state-of-the-art synthesis techniques are already able to mimic biological active filaments with linear chains, composed by active colloidal particles [7-10], often named "active polymers." From a theoretical perspective, while many works on the topic have focused on the collective dynamics of active polar gels [11], actin filaments [12,13], and microtubules [14], recently, single-polymer dynamics has received more attention in diverse scenarios spanning from polymers embedded in a bath of active particles [15-20], flagellated microswimmers [21], and polymers composed of active monomers [22-29]. These works have shown that the details of the coupling between the local active stresses and the conformation of the polymer backbone are crucial for determining the overall dynamics of the polymer. This occurs, for example, in biological processes like DNA-RNA duplication or translation and protein synthesis, where a region of the backbone is under the action of tangential forces induced by active displacement of enzymes.

In this Letter, we characterize the structure and dynamics of a self-avoiding linear polymer composed by axisymmetric active spherical monomers connected by linear springs. In order to highlight the relevance of the orientation of the activity of the monomers with respect to the local conformation of the polymer, we perform Brownian dynamics simulations for different couplings between the local orientation of the active monomers and the conformation of the polymer backbone (Fig. 1). Our results show that, when the direction of the axis of the active monomers is tangent to the local instantaneous conformation of the chain, as it happens for ribosomes and DNA-RNA polymerase or for Janus self-propelled necklaces [9], the activity of the monomers reduces the gyration radius of the polymer that enters in a globularlike state. At the same time, the activity of the monomers promotes the effective diffusion of the polymer, inducing an enhanced diffusion coefficient that eventually becomes essentially independent of the polymer length. These effects are due to the tangential action of the active monomers and disappear when the axis of the active

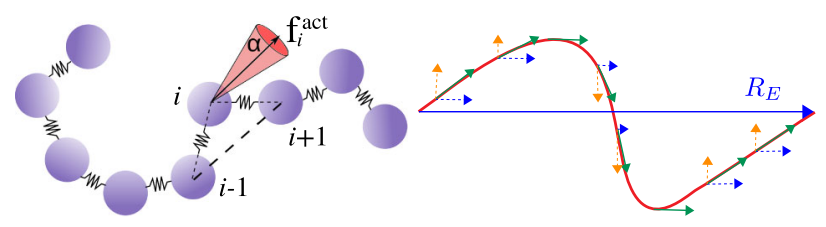

FIG. 1. Schematic sketch of the active polymer. (Left) Polymer regarded as beads connected by linear springs. For each bead $i$ the active force $\mathbf{f}_{i}^{\text {act }}$ diffuses in a cone around the vector $\mathbf{r}_{i+1, i-1}$. The amplitude of the cone is $2 \alpha$. (Right) Continuum representation of the polymer, for $\alpha=0$, with the local self-propulsive force (green arrows) and its components parallel (blue arrows) and perpendicular (orange arrows) to the end-to-end vector $R_{E}$. 

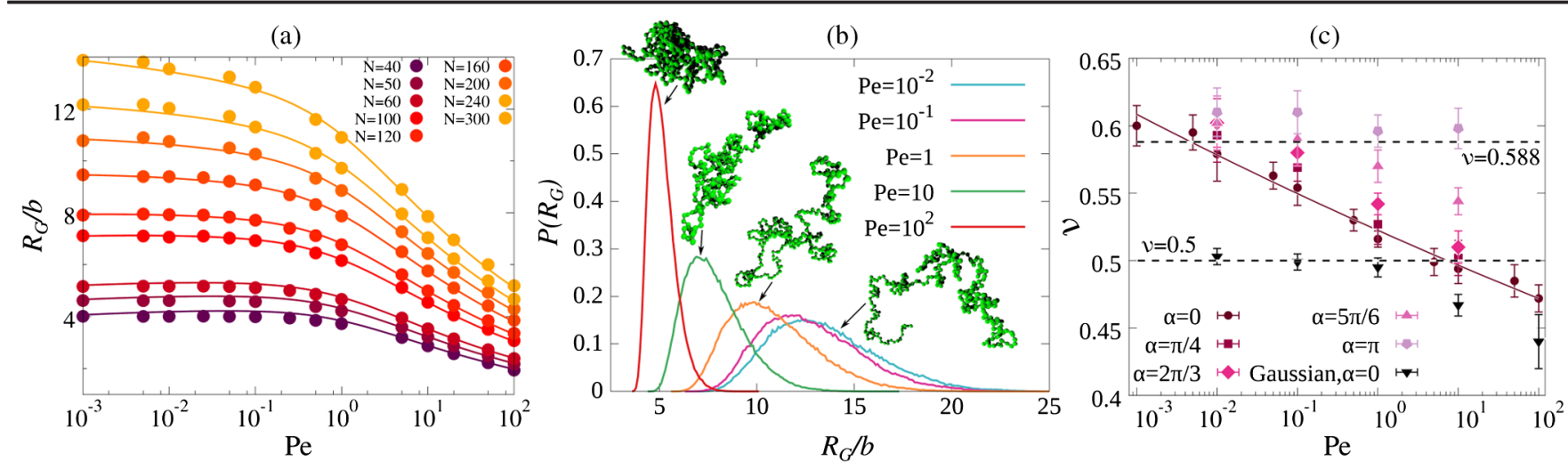

FIG. 2. (a) $R_{G}$ as function of Pe for different polymer sizes $N$. Solid lines are fit of the numerical data with Eq. (3), with $a_{R_{G}}=0.615 \pm 0.003, h_{R_{G}}=0.0265 \pm 0.0007, c_{R_{G}}=0.162 \pm 0.003$, and $\delta_{R_{G}}=0.0005 \pm 0.0002$. (b) Probability distribution $P\left(R_{G}\right)$ of the radius of gyration for a polymer $N=300$, for different values of Pe. Snapshots of representative polymer conformations at different Pe are shown above the curves. (c) Exponent $\nu$ of the gyration radius $R_{G}$, for different values of Pe. Continuous line is a powerlaw fit of $\nu$ for the case $\alpha=0$, given by Eq. (2). Similar power-law scaling holds also for $0<\alpha<\pi$ (and for the Gaussian case for $\mathrm{Pe} \geq 1$ ). Dashed lines show the value of $\nu$ for self-avoiding and Gaussian polymers for $\mathrm{Pe}=0$.

monomers is uncorrelated from the conformation of the polymer. In this latter case, it has been shown that the activity of the monomers acts as an "effective higher temperature" [24]. In order to rationalize our results, we set up a minimal stochastic model that, supported by numerical data, quantitatively captures the dependence of the diffusion coefficient on the controlling parameters.

We model the polymer as a bead-spring self-avoiding chain of $N$ monomers in three dimensions, suspended in an homogeneous fluid. The activity of each monomer $i$ is accounted by a force $\mathbf{f}_{i}^{\text {act }}$, with constant magnitude $f^{\text {act }}$ that can be made dimensionless by introducing the Péclet number $(\mathrm{Pe})[30]$

$$
\mathrm{Pe} \equiv \frac{f^{\text {act }} b}{k_{B} T},
$$

where $b$ is the monomer diameter, $k_{B}$ is the Boltzmann constant, and $T$ is the absolute temperature. We constrain the direction of $\mathbf{f}_{i}^{\text {act }}$ inside a cone with aperture $2 \alpha$, whose main axis is parallel to $\mathbf{r}_{i+1, i-1} \equiv \mathbf{r}_{i+1}-\mathbf{r}_{i-1}$, i.e., the vector connecting the first neighbors of monomer $i$ along the polymer backbone (Fig. 1). Such construction does not apply to the first and last monomers of the chain, which are passive. In particular, when $\alpha=0$, the vector $\mathbf{f}_{i}^{\text {act }}$-in a continuous description of the polymer-is bound to be tangent to the polymer backbone, inducing a strong correlation between the local stresses induced by the activity of the monomers and the local conformation of the chain. In contrast, for $\alpha=\pi$, each force $\mathbf{f}_{i}^{\text {act }}$ is independent of the local conformation of the polymer.

Neighboring monomers along the polymer backbone are held together via a harmonic potential $V_{i}^{(\mathrm{sp})}(r) \equiv$ $\sum_{j=i-1, i+1}\left(K^{(\mathrm{sp})} / 2\right)\left(r_{i, j}-b\right)^{2}$, where $r_{i, j}$ is the distance between the monomers $i$ and $j$. Non-neighboring monomers that are closer then the monomer size $b$ repel each other through a purely repulsive harmonic potential $V_{i}^{m m}(r) \equiv \sum_{j \neq i-1, i+1}\left(K^{(\mathrm{sp})} / 2\right)\left(r_{i, j}-b\right)^{2}$. We fix $K^{(\mathrm{sp})}=$ $100 k_{B} T / b$ to avoid crossing events. We perform Brownian dynamics simulations neglecting hydrodynamic interactions among monomers; i.e., we investigate the "Rouse" regime (see Supplemental Material [31]).

First, we consider the effect of the activity on the global conformation of the chain. In this regard, we compute the radius of gyration $R_{G}$ as function of the Péclet number and the polymer size $N$. Interestingly, for $\alpha=0$, we observe a dramatic decrease of the average value of $R_{G}$ [Fig. 2(a)]; at the same time, the distribution of $R_{G}$ becomes more peaked [Fig. 2(b)], indicating that the chain gets trapped in a crumpled, collapsed state. This behavior, reminiscent of a coil-to-globule transition, can be understood in the limit of $\mathrm{Pe} \ll \beta K^{(\mathrm{sp})} b$, i.e., when the external force is small compared to the interaction among subsequent monomers on the backbone. Looking at the bottom panel of Fig. 1, we notice that the local force perpendicular to the end-to-end vector $R_{E}$ changes sign depending on the curvature of the polymer backbone with respect to the end-to-end vector (orange arrows in the bottom panel of Fig. 1). Hence, for a polymer with almost fixed length, as it is when Pe $\ll \beta K^{(\mathrm{sp})} b$, the net effect of the local force is to promote configurations with larger loops about the end-to-end vector, hence reducing both $R_{E}$ and $R_{G}$. Such a dynamics is visible in the in the Supplemental Videos, where the polymer makes large, smooth curves, which result in a loose bundle, reminiscent of a common yarn ball [34]. In addition, Fig. 2(c) shows that for $\mathrm{Pe}>10^{-2}$ the dependence of the scaling exponent on Pe can be captured by

$$
\nu(\mathrm{Pe})=0.52 \mathrm{Pe}^{-0.022}
$$

i.e., $\nu$ diminishes upon increasing Pe. Using this relation, we have fitted the dependence of $R_{G}$ on Pe via a relatively simple function 


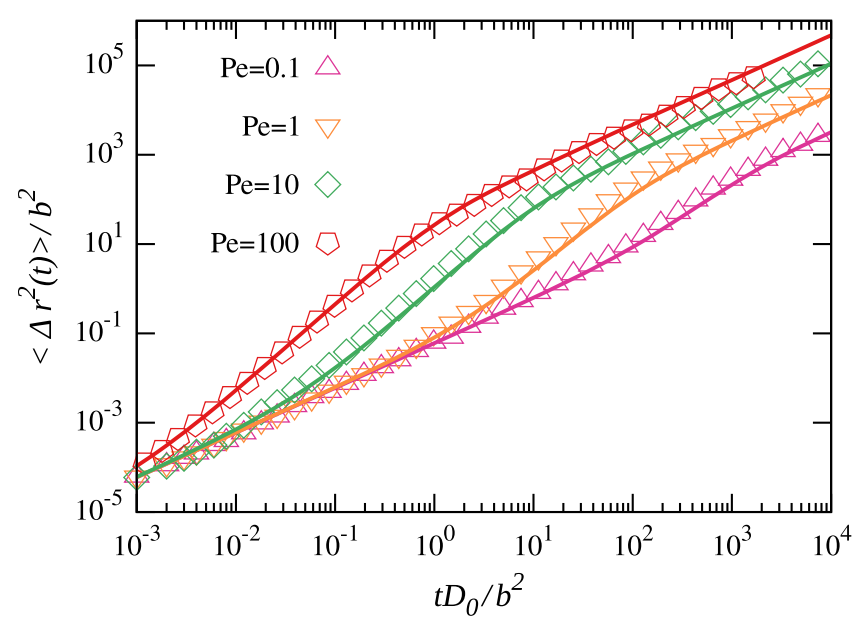

FIG. 3. Mean square displacement $\left\langle\Delta r^{2}(t)\right\rangle$ of the center of mass of the polymer as function of time, for different values of Pe and $N=100$. Symbols are numerical data, lines are the prediction of the model, Eq. (5).

$$
R_{G}=b \frac{a_{R_{G}}+h_{R_{G}} \ln \left(\sqrt{\delta_{R_{G}}^{2}+\mathrm{Pe}^{2}}\right)}{(\mathrm{Pe}+1)^{c_{R_{G}}}} N^{\nu(\mathrm{Pe})},
$$

where $a_{R_{G}}+h_{R_{G}} \ln \left(\sqrt{\delta_{R_{G}}^{2}+\mathrm{Pe}^{2}}\right)$ captures the dependence at small $\mathrm{Pe}$, whereas $1 /(\mathrm{Pe}+1)^{c_{R_{G}}}$ encodes the decay of $R_{G}$ for larger values of Pe. We remark that $a_{R_{G}}, h_{R_{G}}, c_{R_{G}}$, and $\delta_{R_{G}}$ are parameters that are independent of $N$ and Pe; further, Eq. (3), as Eq. (2), is valid only for $\mathrm{Pe}>10^{-2}$.

Remarkably, we observe that a similar phenomenology holds in the case of a Gaussian polymer [black triangles in Fig. 2(c)]. This hints that the activity-induced collapse is not strictly related to self-avoidance. The reduction of $R_{G}$ upon increasing the activity is surprising, since the activity has often been suggested to affect the dynamics as an effective warmer temperature [18,24]. In contrast, our results show the opposite behavior, as the activity leads the polymer towards a globular state, which typically happens upon cooling self-attractive polymers.

Decoupling the direction of the activity from the conformation of the backbone-i.e., considering values $\alpha>0$-mitigates the collapse of the chain, as captured by the reduction of the scaling exponent of $R_{G}$ shown in Fig. 2(c) and Supplemental Material Fig. S1 [31]. The decreasing trend in $\nu$ as function of Pe, previously discussed for $\alpha=0$, still holds for $0<\alpha<\pi$, although with a milder slope as $\alpha$ increases. In particular, for $\alpha=\pi$ we recover the conventional scaling exponent $\nu \sim 0.60 \pm 0.02$ for all the values of Pe, similar to what is shown in Ref. [24]. Hence, for $\alpha=\pi$ the activity does not lead to dramatic changes in the polymer conformation, in contrast to the cases $\alpha \neq \pi$. Next, we consider the effect of the activity on the dynamics, focusing on the mean square displacement of the center of mass of the polymer $\left\langle\left[\mathbf{r}_{\text {c.m. }}(t)-\mathbf{r}_{\text {c.m. }}(0)\right]^{2}\right\rangle \equiv \Delta r^{2}(t)$. When $\alpha=0$, three different regimes in the mean square displacement can be identified (Fig. 3). At very short times, $\tau<\tau_{\text {short }}$,
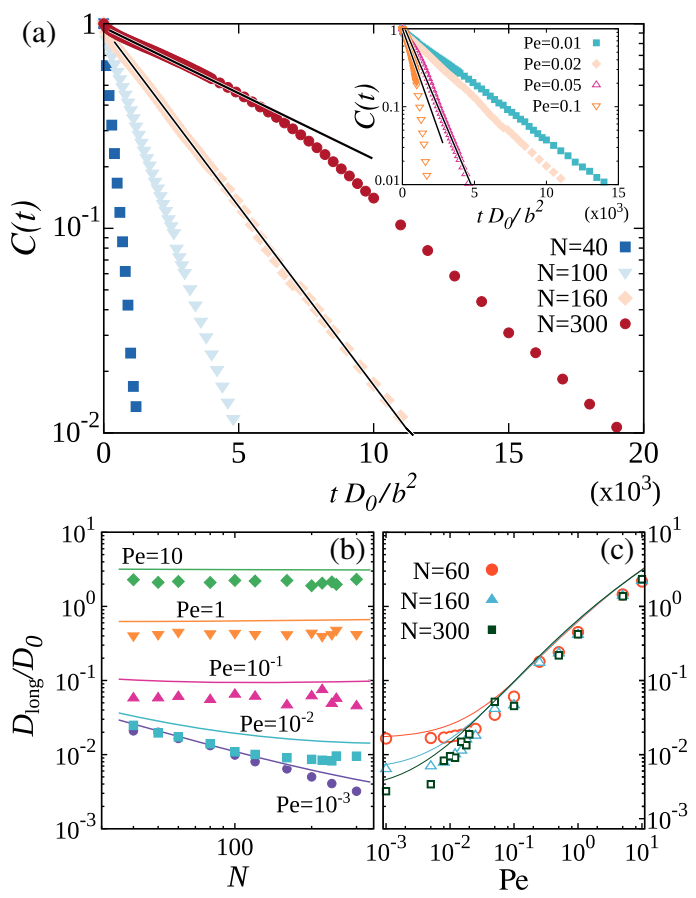

FIG. 4. (a) Time correlation function $C(t)$ of the end-to-end vector for $\mathrm{Pe}=0.02$ and different values of $N$. Black solid lines highlight the exponential decay for lower values of $N$. (Inset) $C(t)$ for $N=160$ and different values of Pe. (b) Translational long-time diffusion coefficient $D_{\text {long }}$ as function of the polymer length $N$, for different values of the Péclet numbers Pe. Data refer to $\alpha=0$. The lines mark the theoretical predictions according to Eq. (6) where $a_{R_{E}}=1.46 \pm 0.02, h_{R_{E}}=0.058 \pm 0.004, c_{R_{E}}=$ $0.201 \pm 0.007$, and $\delta_{R_{E}}=0.0005 \pm 0.0002$ have been determined by fitting the dependence of $R_{E}$ on Pe and $N$. (c) $D_{\text {long }}$ as function of Pe, for different polymer sizes $N$. The lines mark the theoretical prediction given by Eq. (6).

a passive diffusive regime $\left\langle\Delta r^{2}(t)\right\rangle \propto D_{\text {short }} t$ with $D_{\text {short }}=$ $D_{0} / N$ takes place. At intermediate times, $\tau_{\text {short }}<\tau<\tau_{\text {long }}$, a transient superdiffusive regime, typical of active systems [35], is observed. Last, at long times, $\tau>\tau_{\text {long }}$, the diffusive regime is recovered, $\left\langle\Delta r^{2}(t)\right\rangle \propto D_{\text {long }} t$, characterized by an enhanced diffusion coefficient $D_{\text {long }}>D_{\text {short }}$.

Surprisingly, as shown in Figs. 4(b) and 4(c), $D_{\text {long }}$ becomes independent of the polymer size upon increasing Pe. To rationalize the dependence of $D_{\text {long }}$ on $N$ and Pe we regard the center of mass of the polymer as a pointlike particle under the action of an external force $\mathbf{F}^{\text {act }} \equiv$ $\sum_{i=1}^{N} \mathbf{f}_{i}^{\text {act }}$, given by the sum of all the contributions stemming from the monomers. For $\alpha=0$, $\mathbf{F}^{\text {act }}$ is proportional to the end-to-end vector $\mathbf{R}_{E} \equiv \mathbf{r}_{N}-\mathbf{r}_{1}$. Accordingly, $\mathbf{F}^{\text {act }}$ can be regarded as a random force acting on the center of mass with zero average and whose time correlations $C(t)$ are captured by the time correlation of $\mathbf{R}_{E}$

$$
C(t) \equiv\left\langle\mathbf{R}_{E}(t) \cdot \mathbf{R}_{E}(0)\right\rangle .
$$

For a passive polymer $(\mathrm{Pe}=0)$ the function $C(t)$ decays exponentially [36], with a characteristic relaxation time $\tau$. 
In contrast, for Pe $\neq 0$, Fig. 4(a) shows that the exponential decay holds reasonably well within the range of explored values of $N$ and Pe, although for large values of $N$ and Pe a deviation from such a behavior is observed [red circles in Fig. 4(a) and orange triangles in the inset]. The behavior of $C(t)$ in such regimes is consistent with a compressed exponential decay, similar to what has been observed in soft-glass and out-of-equilibrium materials [37], where it is due to a long-range persistent Gaussian noise [38]. This phenomenon goes beyond the scope of the present Letter and will be discussed in future works. The model proposed provides an analytical solution for the mean square displacement of the center of mass of the polymer is (see Supplemental Material for a detailed derivation [31])

$$
\begin{aligned}
& \left\langle\mathbf{r}_{\text {c.m. }}^{2}(t)\right\rangle_{\xi, \eta} \\
& =2\left(\frac{D_{0} \tau_{0}}{b^{2}} \mathrm{Pe} \frac{\left[a_{R_{E}}+h_{R_{E}} \ln \left(\sqrt{\delta_{R_{E}}^{2}+\mathrm{Pe}^{2}}\right)\right]^{2}}{(\mathrm{Pe}+1)^{2 c_{R_{E}}}} N^{2 \nu(\mathrm{Pe})-1}\right) \\
& \quad \times D_{0}\left(t+\tau e^{-t / \tau}-\tau\right)+2 d \frac{D_{0}}{N} t
\end{aligned}
$$

where $a_{R_{E}}, h_{R_{E}}, c_{R_{E}}$, and $\delta_{R_{E}}$ are fitting parameters that are independent of $N$ and Pe [see Fig. S8(b) and Eq. (6) of the Supplemental Material [31]], $d$ is the dimensionality of the system, and $\nu(\mathrm{Pe})$ is the scaling exponent of $R_{E}$ [Fig. S8(a)]. The first (second) term in Eq. (5) represents the contribution to the diffusion of the center of mass due to activity (thermal fluctuations). Remarkably, Eq. (5) quantitatively agrees with the numerical data with no additional fitting parameters [39]. The super- and active-diffusive regimes can be identified as the short- and long-time limits of the "active" contribution in Eq. (5). The crossover times $\tau_{\text {short }}$ and $\tau_{\text {long }}$ are predicted by comparing the leading contributions at short and long times, respectively (see Supplemental Material [31]). These predictions agree relatively well with the numerical data [see Figs. S7(a) and S7(b)]. In particular, $\tau_{\text {long }} \propto N /$ Pe, i.e., $\tau_{\text {long }}$ scales as the decorrelation time of the end-to-end vector and $\tau_{\text {short }} \propto$ $\mathrm{Pe}^{-1.6}$ (from numerical data $\tau_{\text {short }} \propto \mathrm{Pe}^{-1.5}$ ); i.e., $\tau_{\text {short }}$ is predicted to be independent of the polymer size. From Eq. (5), the long-time diffusion coefficient $D_{\text {long }}$ can be calculated

$$
\begin{aligned}
D_{\text {long }}= & D_{0}\left(\frac{D_{0} \tau_{0}}{b^{2}} \frac{\mathrm{Pe}}{d} \frac{\left[a_{R_{E}}+h_{R_{E}} \ln \left(\sqrt{\delta_{R_{E}}^{2}+\mathrm{Pe}^{2}}\right)\right]^{2}}{(\mathrm{Pe}+1)^{2 c_{R_{E}}}}\right. \\
& \left.\times N^{2 \nu(\mathrm{Pe})-1}+\frac{1}{N}\right) .
\end{aligned}
$$

In particular, Eq. (6) shows that for large values of Pe or $N$ the thermal contribution (second term in the brackets) can be disregarded and $D_{\text {long }}$ becomes essentially

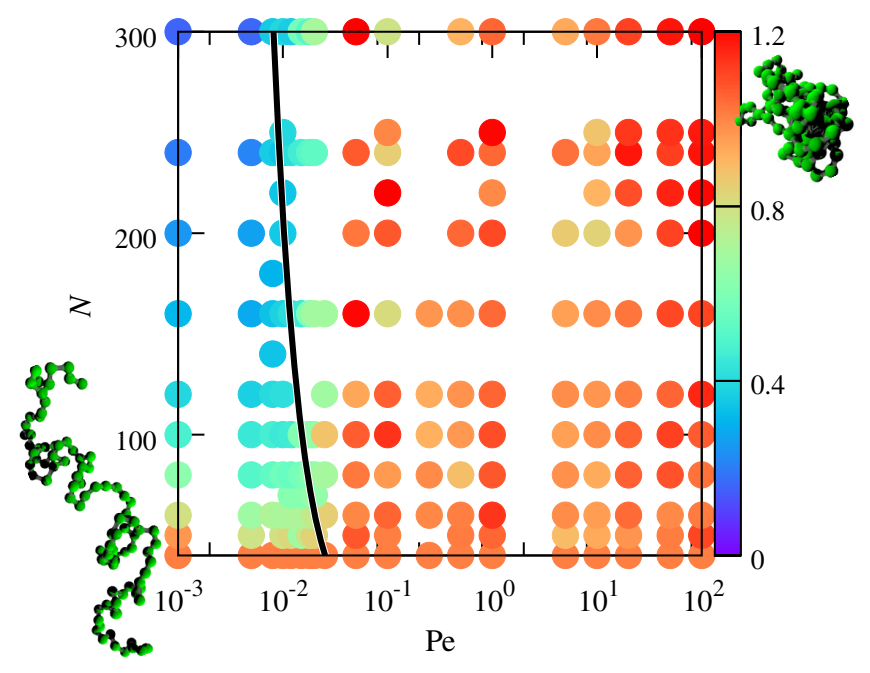

FIG. 5. Normalized diffusion coefficient $\tilde{D} \equiv D_{\text {long }}(N, \mathrm{Pe}) /$ $D_{\text {long }}(40, \mathrm{Pe})$ (color coded) as function of $N$ and Pe. The solid line represents the locus where the two contributions in Eq. (6) are equal.

independent of $N$ since $2 \nu(\mathrm{Pe})-1 \ll 1$ [40]. Interestingly, as shown in Figs. 4(b) and 4(c), our model captures quantitatively the asymptotic growth of $D_{\text {long }}$ upon increasing Pe, whereas it predicts a smoother transition to the passive regime with respect to the numerical results.

Equating the two contributions on the rhs of Eq. (6) allows us to identify two regions in a $\mathrm{Pe}-N$ phase diagram, namely, a region where $D_{\text {long }} \propto 1 / N$ and a region in which $D_{\text {long }}$ is almost $N$ independent. Figure 5 shows the diffusion coefficient $D_{\text {long }}(N, \mathrm{Pe})$ normalized by the value obtained for $N=40$, for each value of Pe. Accordingly, the color code in Fig. 5 indicates the region where $D_{\text {long }}$ decreases with the polymer size (blue region) and the region where $D_{\text {long }}$ is almost independent of $N$ (red region). The transition between the two regions, marked by the solid line in Fig. 5, is properly captured by our theoretical model. Moving from the lower to the upper region of the Pe- $N$ phase diagram (Fig. 5), the radius of gyration and its fluctuations decrease [Fig. 2(a)], and the continuous coil-to-globulelike transition described before can be observed. For $0<\alpha<\pi$, a similar phenomenology is observed (Fig. S5), with a reduced dependence of $D_{\text {long }}$ on Pe. In the limiting case $\alpha=\pi$, for which the local activity of the monomer is uncorrelated from the structure of the polymer, a passivelike behavior is recovered, $D_{\text {long }} \sim \xi D_{0} / N$, where $\xi>1$ is a prefactor that depends on Pe and marks the active nature of the system. In order to address the role of self-avoidance in the aforementioned dynamics, we have performed similar numerical simulations for Gaussian polymers (Fig. S6). We found qualitatively similar results, although with a weaker dependence of $D_{\text {long }}$ upon $N$ and Pe. Such a reduced sensitivity is expected since $\mathbf{F}^{\text {act }} \propto \mathbf{R}_{E}$ that, for a Gaussian polymer, scales with a smaller exponent as compared with a 
self-avoiding one [Fig. 2(b)]. In conclusion, we have studied the dynamics of an active polymer in three dimensions. We have shown that both the conformation and the diffusion of the polymer are strongly affected by the activity of the monomers. In particular, the effect of the activity is strongest when it is bound to be tangent to the backbone of the polymer $(\alpha=0)$ and it smoothly reduces upon releasing such a constraint (i.e., increasing $\alpha$ ). Concerning the polymer conformation, we found that, when the activity dominates over the thermal motion, the polymer undergoes a coil-to-globulelike transition as captured by the decrease of the scaling exponent of the gyration radius (Fig. 2); i.e., increasing the activity is analogous to reducing the temperature for self-attracting polymers. At the same time, the diffusion coefficient of the polymer becomes independent of its size and it becomes, possibly, several orders of magnitude larger than the corresponding equilibrium value. In this latter respect, the activity acts as a higher temperature that enhances the diffusion. These results might inspire the realization of highly mobile drug delivery carriers, where the drugs are embedded along the backbone of active polymers, whose active monomers have their axis of motion aligned with the polymer backbone. Interestingly, current state-of-the-art techniques may open up the possibility to synthesize active polymers by means of surface-shell functionalization of single colloids [41-43].

We acknowledge I. Coluzza, C. Dellago, C. N. Likos, and L. Rovigatti for helpful discussions. V. B. acknowledges the support from the Austrian Science Fund (FWF), Grant No. M 2150-N36, and from the European Commission through the Marie Skłodowska-Curie Fellowship No. 748170 ProFrost. The computational results presented have been achieved using the Vienna Scientific Cluster (VSC).

*vbianco283@gmail.com

"emanuele.locatelli@univie.ac.at *malgaretti@is.mpg.de

[1] B. Alberts, J. Alexander, L. Julian, R. Martin, R. Keith, and W. Peter, Molecular Biology of the Cell (Garland Science, New York, 2002).

[2] R. K. P. Zia, J. J. Dong, and B. Schmittmann, J. Stat. Phys. 144, 405 (2011).

[3] J. Wang and W. Gao, ACS Nano 6, 5745 (2012).

[4] K. K. Dey, X. Zhao, B. M. Tansi, W. J. Mendez-Ortiz, U. M. Cordova-Figueroa, R. Golestanian, and A. Sen, Nano Lett. 15, 8311 (2015).

[5] M. Medina-Sànchez, L. Schwarz, A. K. Meyer, F. Hebenstreit, and O. G. Schmidt, Nano Lett. 16, 555 (2016).

[6] J. Simmchen, J. Katuri, W. E. Uspal, M. N. Popescu, M. Tasinkevych, and S. Sánchez, Nat. Commun. 7, 10598 (2016).

[7] R. Dreyfus, J. Baudry, M. L. Roper, M. Fermigier, H. A. Stone, and J. Bibette, Nature (London) 437, 862 (2005).

[8] L. J. Hill, N. E. Richey, Y. Sung, P. T. Dirlam, J. J. Griebel, E. Lavoie-Higgins, I.-B. Shim, N. Pinna, M.-G. Willinger,
W. Vogel, J. J. Benkoski, K. Char, and J. Pyun, ACS Nano 8, 3272 (2014).

[9] B. Biswas, R. K. Manna, A. Laskar, P. B. Sunil Kumar, R. Adhikari, and G. Kumaraswamy, ACS Nano 11, 10025 (2017).

[10] D. Nishiguchi, J. Iwasawa, H.-R. Jiang, and M. Sano, New J. Phys. 20, 015002 (2018).

[11] M. C. Marchetti, J. F. Joanny, S. Ramaswamy, T. B. Liverpool, J. Prost, M. Rao, and R. A. Simha, Rev. Mod. Phys. 85, 1143 (2013).

[12] M. Bathe, C. Heussinger, M. M. Claessens, A. R. Bausch, and E. Frey, Biophys. J. 94, 2955 (2008).

[13] V. Schaller, C. Weber, C. Semmrich, E. Frey, and A. R. Bausch, Nature (London) 467, 73 (2010).

[14] F. Ndlec, T. Surrey, A. C. Maggs, and S. Leibler, Nature (London) 389, 305 (1997).

[15] A. Kaiser and H. Löwen, J. Chem. Phys. 141, 044903 (2014).

[16] J. Harder, C. Valeriani, and A. Cacciuto, Phys. Rev. E 90, 062312 (2014).

[17] J. Shin, A. G. Cherstvy, W. K. Kim, and R. Metzler, New J. Phys. 17, 113008 (2015).

[18] H. Vandebroek and C. Vanderzande, Phys. Rev. E 92, 060601 (2015).

[19] T. Eisenstecken, G. Gompper, and R. Winkler, Polymers 8, 304 (2016).

[20] N. Samanta and R. Chakrabarti, J. Phys. A 49, 195601 (2016).

[21] J. Elgeti, R. G. Winkler, and G. Gompper, Rep. Prog. Phys. 78, 056601 (2015).

[22] A. Ghosh and N. Gov, Biophys. J. 107, 1065 (2014).

[23] R. Chelakkot, A. Gopinath, L. Mahadevan, and M. F. Hagan, J. R. Soc. Interface 11, 20130884 (2014).

[24] A. Kaiser, S. Babel, B. ten Hagen, C. von Ferber, and H. Löwen, J. Chem. Phys. 142, 124905 (2015).

[25] R. E. Isele-Holder, J. Elgeti, and G. Gompper, Soft Matter 11, 7181 (2015).

[26] R. E. Isele-Holder, J. Jäger, G. Saggiorato, J. Elgeti, and G. Gompper, Soft Matter 12, 8495 (2016).

[27] D. Osmanović and Y. Rabin, Soft Matter 13, 963 (2017).

[28] R. G. Winkler, J. Elgeti, and G. Gompper, J. Phys. Soc. Jpn. 86, 101014 (2017).

[29] S. Gonzalez and R. Soto, New J. Phys. 20, 053014 (2018).

[30] Via the Stokes-Einstein relation, $D_{0}=k_{B} T / \zeta$, with $\zeta$ the friction coefficient of the monomer and using $\zeta v^{\text {act }} \equiv f^{\text {act }}$, the Péclet number in Eq. (1) can be reduced to its common form: $\mathrm{Pe}=v^{\text {act }} b / D_{0}$.

[31] See Supplemental Material at http://link.aps.org/ supplemental/10.1103/PhysRevLett.121.217802 for a sample video, additional numerical data, and detailed theoretical calculations, including Refs. [32,33].

[32] G. Marsaglia, Comput. Math. Appl. 12, 1187 (1986).

[33] M. Fixman, J. Chem. Phys. 36, 306 (1962).

[34] This phenomenon is emphasized by the distribution of the bending angles formed by three consecutive monomers (see Fig. S3). Upon increasing Pe, the probability of smaller bending angles increases, implying that the polymer is locally more straight.

[35] M. E. Cates, Rep. Prog. Phys. 75, 042601 (2012). 
[36] M. Doi and S. Edwards, The Theory of Polymer Dynamics, International Series of Monographs on Physics (Clarendon Press, Oxford, 1986).

[37] J. Gabriel, T. Blochowicz, and B. Stühn, J. Chem. Phys. 142, 104902 (2015).

[38] J.-P. Bouchaud, in Anomalous Transport (Wiley-VCH Verlag $\mathrm{GmbH} \& \mathrm{Co}$. KGaA, Weinheim, 2008), pp. 327-345.

[39] $a_{R_{E}}, h_{R_{E}}$, and $c_{R_{E}}$ are obtained by fitting the dependence of $R_{E}$ on Pe and $N$ [see Fig. S8(b)].
[40] For example, for $\mathrm{Pe}=1$, the term $N^{2 \nu(\mathrm{Pe})-1}$ changes by a factor 2 while changing $N$ by 3 orders of magnitude, from $N=10^{2}$ to $N=10^{5}$.

[41] Y. Liu, K. Li, J. Pan, B. Liu, and S.-S. Feng, Biomaterials 31, 330 (2010).

[42] S. A. J. van der Meulen and M. E. Leunissen, J. Am. Chem. Soc. 135, 15129 (2013).

[43] L. Feng, L.-L. Pontani, R. Dreyfus, P. Chaikin, and J. Brujic, Soft Matter 9, 9816 (2013). 